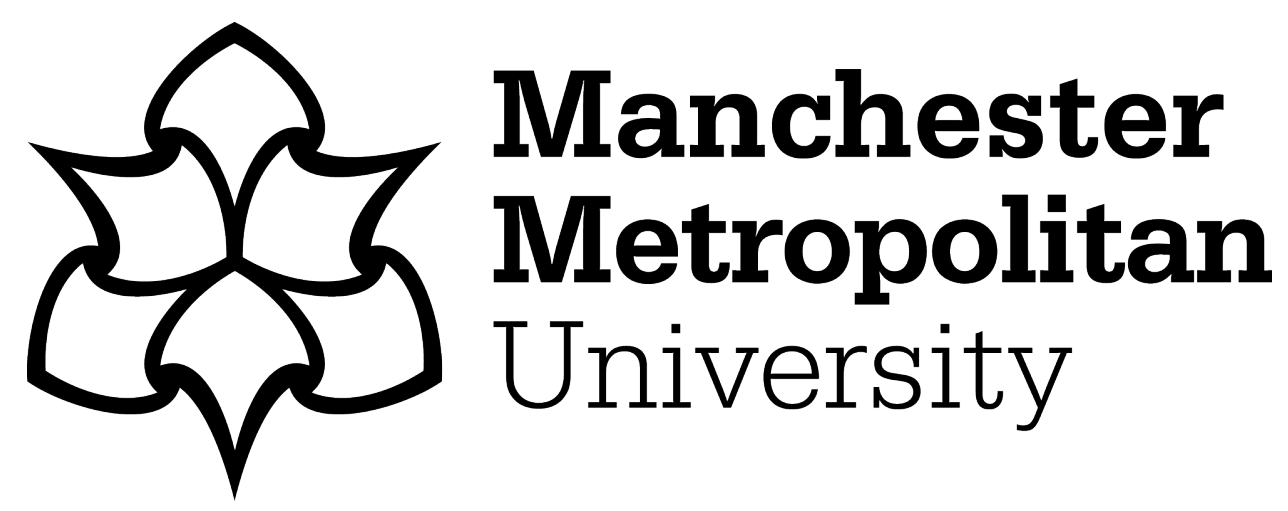

Li, J, Wu, J, Li, C, Yang, W, Bashir, AK, Li, J and Al-Otaibi, YD (2022) Information-Centric Wireless Sensor Networking Scheme with Water-DepthAwareness Content Caching for Underwater IOT. IEEE Internet of Things Journal, 9 (2). pp. 858-867. ISSN 2327-4662

Downloaded from: https://e-space.mmu.ac.uk/627679/

Version: Accepted Version

Publisher: Institute of Electrical and Electronics Engineers (IEEE)

DOI: https://doi.org/10.1109/JIOT.2021.3058272

Please cite the published version 


\title{
Information-Centric Wireless Sensor Networking Scheme with Water-Depth-Awareness Content Caching for Underwater IoT
}

\author{
Jiana Li, Jun Wu, Changlian Li, Wu Yang, Ali Kashif Bashir, Jianhua Li, and Yasser D. Al-Otaibi
}

\begin{abstract}
The existing underwater Internet of Things (UIoT) is based on IP architecture, which is not conducive to the efficient storage and distribution of huge amounts of content generated in underwater. Actively pushing all content to users causes much unnecessary resource consumption in the UIoT. The information centric networking (ICN) architecture opens new horizons up for these challenges. However, the slowness of underwater propagation speed makes traditional ICN not suitable for UIoT, especially considering about delay time. In this paper, we propose an information-centric wireless sensor networking scheme with water-depth-aware content caching (ICWSN-WDA) to solve the above challenges. First, we design a naming scheme and a hybrid communication mode suitable for ICWSN-WDA. The communication mode in underwater we design is divided into push and pull traffic, which balances energy consumption and delay time. Secondly, we define a push level to decide which water depth the content actively pushes to, finding a suitable junction point of two modes. Thirdly, as the water depth is deeper, it becomes more difficult to replace sensor battery. To save energy consumption of deep-water sensors, water-depth-aware caching mechanism is proposed based on water depth, popularity and senor energy. Our extensive evaluation confirms the effectiveness of our proposed scheme, and it balances energy consumption constraints and latency.
\end{abstract}

Index Terms-Underwater Internet of Things (UIoT), Information-Centric Networking (ICN), caching, water-depthawareness.

\section{INTRODUCTION}

$\mathbf{O}$ VER $70 \%$ of the earth is made up of oceans. The process of ocean exploration has never stopped. Ocean has always been energy, nutrition production, natural resources and

This work is sponsored by the National Natural Science Foundation of China (Grant No. 61831007 and 61972255). (Corresponding author: Jun Wu and Wu Yang.)

Jianhua Li, Jun Wu and Jiana Li are with Shanghai Key Laboratory of Integrated Administration Technologies for Information Security, School of Cyber Security, Shanghai Jiaotong University, Shanghai 200240, China.(email: junwuhn@sjtu.edu.cn)

Changlian $\mathrm{Li}$ is with China Information Consulting \& Designing Institute Co., Ltd., China. (e-mail: lichl1@ chinaunicom.cn)

Wu Yang is with the Information Security Research Center, Harbin Engineering University, Harbin 150001, China. (e-mail: yangwu@ hrbeu.edu.cn)

Ali Kashif Bashir is with Department of Computing and Mathematics, Manchester Metropolitan University, UK, and School of Electrical Engineering and Computer Science, National University of Science and Technology, Islamabad (NUST), Islamabad, Pakistan.

Yasser D. Al-Otaibi is with department of Information Systems, Faculty of Computing and Information Technology in Rabigh, King Abdulaziz University, Jeddah 21589, Saudi Arabia (e-mail: yalotaibi@kau.edu.sa).

Copyright (c) 20xx IEEE. Personal use of this material is permitted. However, permission to use this material for any other purposes must be obtained from the IEEE by sending a request to pubs-permissions@ieee.org. the main energy in the near future [1]. Underwater communication technology and network equipment, such as automatic underwater vehicles (AUV), underwater towing sensors and automatic robots, have developed rapidly. Therefore, realtime communication between underwater sensors and control centers has been achieved. The establishment of an underwater wireless sensor network facilities long-term surveillance about selected marine areas and the collection of various marine categories of data.

In the underwater Internet of Things (UIoT), data is often transmitted from sensors in the deep water to users in the shallow. The underwater propagation speed is five orders of magnitude lower than the radio channel [2], resulting in a significantly higher delay than traditional networks. Compared with traditional Internet hosts, most underwater sensor devices have a limitation of battery power, memory, and computing ability, as well as expressing mobility. In addition, unlike the case where Internet content is usually large and stable files, Underwater data usually has characteristics of small and constantly changing, and its amount is massive.

UIoT has been detecting and delivering content, and water depth is a feature that other networks and environments do not have. The existing UIoT is based on IP architecture. It cannot store and distribute huge amounts of content efficiently. Nodes in UIoT automatically transmit all collected content and most content transferred is not needed by users, which consumes a lot of energy. This situation contradicts the limited power of sensors. In addition, the topology of UIoT changes as the underwater environment changes, which causes mobility issues. Because of the information-centric network (ICN)'s [3] easy data retrieval and sharing, intra-network caching, content-based name routing and other benefits, ICN can be used as a component of UIoT to improve above problems and provide new possibilities for UIoT. In ICN, each data has its own name. Data can be requested by the specific name, regardless of its location, so billions of devices and huge content can be combined. In particular, caching is a vital part that affects the performance of networks, which can speed up information retrieval and improve latency.This also implies that the ocean data that consumers pay attention to can be selectively cached in the network, avoiding to transfer all data and reducing unnecessary energy consumption of nodes. The ICN node replaces identifying node with identifying content, and separates the location of the content from its name, making UIoT get rid of the mobility problem.

When the existing UIoT which based on IP network 
is converted to information-centric underwater network, the new information-centric UIoT needs to adapt to underwater characteristics without affecting the superiority of the ICN. Considering that the information in the traditional UIoT is unconditionally pushed to users, the traditional ICN's content request and send mode is different from underwater network communication mode. Besides, due to the data transfer environment which information transmits from deep water to shallow water, the limit of equipment resources and the different data concerns of marine users in different marine areas, the traditional internet-used caching algorithm [4] is unsuitable for underwater networks. Underwater informationcentric architecture which can efficiently process content is still an open issue. In order to fill this gap, we make the contributions as follows:

- An information-centric wireless sensor networking scheme with water-depth-aware content caching (ICWSN-WDA) is proposed to send and receive content more efficiently in underwater. Then we design a naming scheme and a hybrid communication mode which divided into push and pull traffic suitable for this scheme.

- We define a push level to decide which water depth the content actively pushes to. It comprehensively takes water depths, popularity and sensor energy into account. The push level finds a suitable junction point of the push and pull modes, balancing delay time and energy consumption and providing a basis for the judgment of the subsequent caching scheme.

- We propose a water-depth-aware content caching mechanism. It can be divided into push-caching and pull caching two caching strategies corresponding to different communication modes. Push-caching calculates cache probability at each sensor by assessing the depth of the content-generated sensor, the current sensor and surrounding sensors. Pull-caching probability considers the above water depth, sensor energy and the popularity of the content.

The remainder of the paper is organized as follows. Section II discusses related work. The proposed ICWSN-WDA are described in Section III. And Section IV shows the detail of water-depth-aware caching in ICWSN-WDA, whereas Section $\mathrm{V}$ presents the evaluation model and results. Section VI concludes the paper.

\section{RELATED WORK}

This section is segmented into two categories: (1) Internet of Things, and (2) applications of Information-centric networks.

\section{A. Internet of Things (IoT) and UIoT}

IoT has gradually integrated into our lives and is often used to collect big data. Moreover, existing advanced technology further improves IoT. [5] realized dynamic management and configuration consensus in blockchain of IoT. Authors of [6] proposed a dynamic detection based on a micro-blockchain structure for vehicles to everything. In order to balance computing resource space-time dynamic demands in smart city [7], a Peer-to-Peer computing resource trading system is designed.
Energy function virtualization of smart grid [8] is used to accelerate the deployment and testing of energy functions. A novel wireless powered edge smart framework [9] solved the problem about the limited battery capacities of IoT devices and the high energy requirements in learning.

The application of IoT has gradually extended to underwater. To promote the development of underwater internet of things (UIoT), it is desirable to have a universal underwater network architecture. [10] provided an overview of the UIoT including proposed network architectures, existing applications and challenges. Inspired by novel softwaredefined techniques and paradigms, [11] pointed that users can customize service-oriented UIoT in the future. The purpose of [2] is to investigate existing network technologies and their applicability in underwater acoustic channels. [12] considered that gliders are regarded as special sensor nodes in the current UIoT and proposed a glider-assist routing scheme to improve the connectivity of the hybrid network. Vinayak Khajuria et al. introduced 3D- underwater wireless sensor networks [13]. The nodes are deployed in groups. In each cluster, there is an anchor node, which receives information from other nodes in the cluster. These groups are at different depth levels.

\section{B. Applications of Information-centric Networks}

Several major ICN architectures have been proposed, such as data oriented network framework, content-centric networking (CCN) [14], publish-subscribe communication mode [15], mobility first (MF) [16] and green ICN [17]. The obvious advantages of ICN make itself a promising networking model for scenarios such as the Internet of Things [18], connected vehicles, smart grids and so on. [19] proposed an advanced node architecture based on NDN. The three-layer NDN architecture supports the Internet of Things, consisting of an application layer, an NDN layer, and an Internet of Things layer. Reza et.al [20] proposed an information-centric network architecture for smart grids that meets their communication requirements. In [21], a fog computing- enabled cognitive network functions virtualization approach is proposed to address smart analysis and the control of computational and storage resources. To improve content naming, addressing, data aggregation, and mobility of IVC, a vehicular information network architecture [22] is designed. [23] focused on security services in the information-centric social network and proposed a fogcomputing-based method for content-aware filtering.

Underwater environment has its own unique characteristics and different application requirements, so the above-mentioned architectures combined with ICN for different environments are unsuitable to be applied to UIoT directly. As far as we know, an information-centric underwater sensor network architecture is still an open issue. In this paper, we propose a innovative information-centric internet of underwater things scheme which fills this void.

\section{The Proposed ICWSN-WDA ARChITECTURE}

\section{A. Overview of ICWSN-WDA}

ICWSN-WDA provides a possibility for the combination of UIoT and ICN. Underwater networks can be described as 
long delays, prone to interruption and sensors with limited resources. The content transmitted by the underwater network is often numerous and complex. Most users of the underwater network are more interested in only specific content, while existing underwater networks send all generated content actively without request.Therefore, the advantages of ICN can make the underwater network play its role better, especially publish/subscribe paradigm and caching mechanism. Thanks to the differences in underwater networks and the factor of water depth, the existing ICN cannot meet the requirements of underwater networks, and the ICN must be optimized to support such restrictions. In this article, we designed three main modules: naming mechanism, communication mode, and caching mechanism.

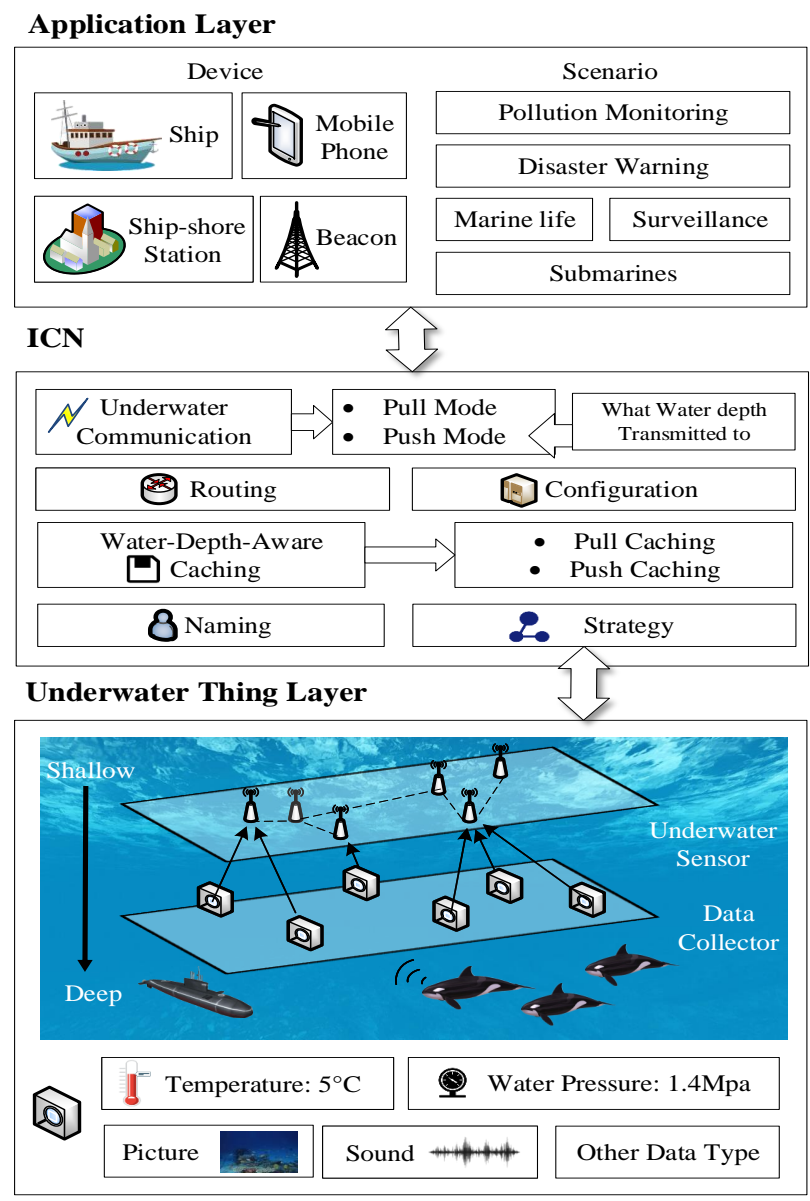

Fig. 1: Overview of ICWSN-WDA scheme

To this end, Fig. 1 shows the architecture of ICWSN-WDA. At the bottom of the architecture, we placed the thing layer, which illustrates many sensors in the underwater network. They can collect various types of data from the ocean and exhibit different constraints (size, battery, cost, processing, storage). And they are at different depths in the ocean. The top of the stack summarizes some representative underwater network applications whose needs should be met. In such an architecture, ICN acts as a network layer and is expected to implement a content-centric underwater network by adapting its modules to its functions.
The ICN layer includes underwater communication, routing, configuration, strategy and caching. ICWSN-WDA needs to operate based on the names of interest packets and data packets. The unstable underwater environment (such as AUV, ocean waves, tides, etc.) The unstable underwater environment makes underwater data transmission prone to severe path loss and route instability. Therefore, it is necessary to design a routing scheme suitable for underwater to obtain better performance [23]. The basic configuration of the network should also be designed according to the specific underwater conditions. Communication is the key part of ICWSN-WDA. Since the underwater acoustic modem is a suitable choice of the current technology for underwater communication [25] [27], the data transmission speed of underwater is significantly lower than the radio propagation speed. Therefore, the publish and subscribe communication mode of the classic informationcentric networking is useless for ICWSN-WDA, and a mixed communication mode of pull and push is designed to balance the limitations and advantages of ICN and UIoT's communication mode. The details of communication mode will be introduced in Section III: C. The strategy mainly refers to the forwarding strategy. The forwarding of interest packets should ensure that the required content can be retrieved, and the forwarding of data packets should make the retrieval time required for users to receive the data packets as short as possible. The strategy also needs to consider the mobility of underwater nodes and the data propagation speed of underwater. A unique hybrid communication mode is designed in ICWSN-WDA, so the caching in ICWSN-WDA should adapt to this communication mode. That is, the caching is divided into push and pull strategies, corresponding to push traffic and pull traffic. At the same time, comprehensively considering the characteristics of underwater sensors, data and underwater depth will optimize caching algorithms.

\section{B. Naming Scheme}

In underwater networks, the naming scheme must accurately describe what the sensors get. Therefore, naming should enable users to fully express the information they need and sensors to accurately describe their data. The data names we design include the following attributes:

- Communication mode. ICWSN-WDA contains push and pull communication modes, where 1 is push traffic and 0 is pull traffic.

- Data type. The name of the data detected by the sensor or required by the user, such as pressure, temperature, humidity, pictures, etc.

- Location. The sensor detects the identifier of the geographic area of the data, usually using the GPS coordinates of the area. Since the depth at water is an important determining factor of the spatial position and the influence factor of the underwater network, it is not included in the name of this place.

- Water Depth. The depth of the ocean where the sensor is located is represented by depth data relative to sea level.

- Detection time. The time period during which the sensor acquired data. It can be a real-time value, or an average over a given time interval. 
- Popularity. Number of times each data type in different ocean areas was requested, initialized to 0 .

ICN's common naming scheme can easily match all of the above attributes. Specifically, we assume the following name structure: "communication mode" / "data type" / "Location" / "depth" / "detection time" / "popularity". A temperature data can be named as shown in Fig. 2. It states that it is in pull mode and carries a temperature measurement value that was detected at the time indicated by the timestamp in a region of longitude 143, latitude 38.4 and depth $122 \mathrm{~m}$, and this area of data has been requested twice.

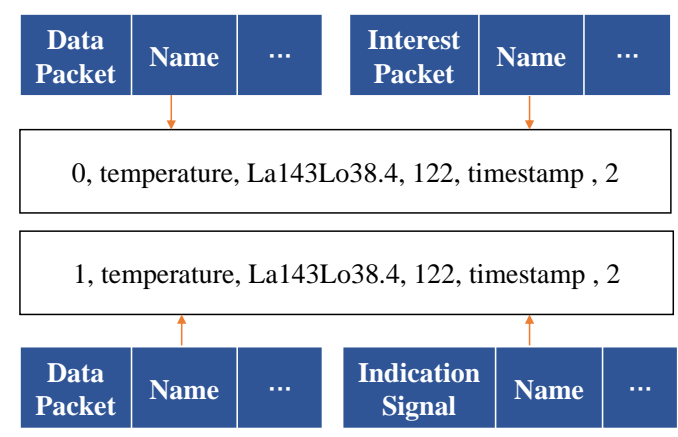

Fig. 2: Examples of packet name

Data packets exist in both push and pull traffic. The communication mode of interest packet is 0 , considering that the interest packet only transmits in pull traffic. The indication signal informs the lower-level sensor that specific content is requested, so its communication mode is determined to be 1 . Besides, the rest structure of the indication signal is similar to the interest packet.

\section{Communication mode}

The traditional ICN network defines a simple and powerful user-driven communication model based on the exchange of interest and data packets. Each data packet carries a hierarchical, specific content name. The depth in the ocean cannot be predicted, and the data transmission speed is also slower than on the ground. It is not advisable to directly apply the traditional ICN in the UIoT, there are two reasons: i) the memory of the underlying sensor is low, and when a particular sensor receives an interest packet, the data that the user requests may be overwritten; ii) The deeper the producer, the longer the retrieval time. The data transmitted from the underlying sensor to users has a longer time delay. ICN can't take its own advantage.

The underwater environment is complex and constantly changing, and generates a large amount of content [29], so the existing underwater network is usually delivered by the producer in real time and actively to the user. The amount of underwater data is inestimable. In reality, most of the content obtained by sensors is not needed by users. If this part of content is directly delivered to users, it will obviously waste a lot of resources. Therefore, it is necessary to balance the limitations and advantages of these two networks' communication mode and we have redesigned the communication mode in ICWSN-WDA.

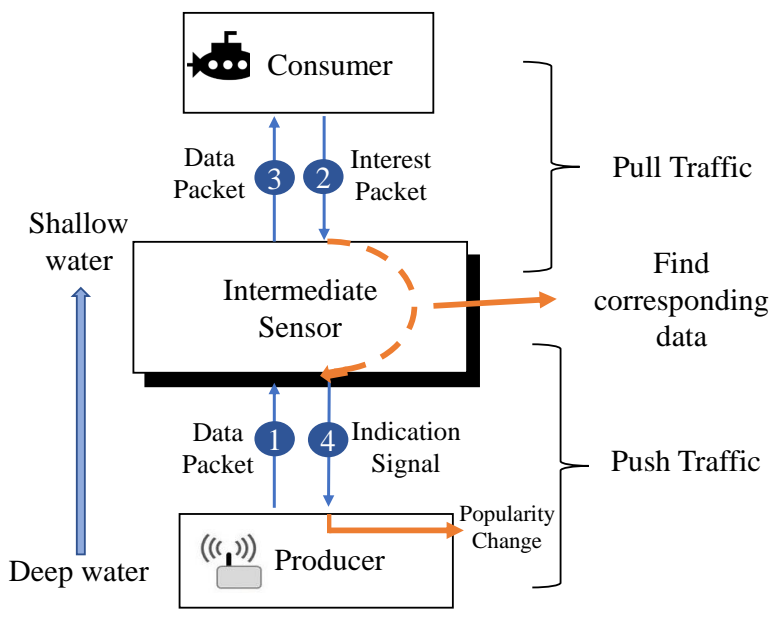

Fig. 3: Two modes in communication process

Deeper places in the ocean are often unreachable and only sensors collect data. In fact, users (receivers) in the ocean are in shallower areas or on the sea surface, so there is no user request data in deep-water areas. Therefore, as proposed communication mode shown in the Fig. 3, after the bottom sensor collects the data, it still directly transmits the data, especially only forwards it to the shallower water. However, it is not directly delivered to the user. This process is stopped at a middle node. The above process is called push traffic. When users request content, they are directly transferred from the node that caches the required content, and there is no need to transmit the interest packet to the underlying producer. This process is called pull traffic. When the node is sending the data packet that the user needs, it also sends an indication signal to the producer. It explains to the producer that this type of data is requested.

\section{WATER-DePth-Aware CONTEnt CACHING}

Underwater sensors at different depths in underwater networks often have large differences, and the contents generated in the deep ocean usually need to be transmitted to shallower water where users exist. The water depth of producers, nodes, etc. all have a greater impact on the transmission which needs to be considered. Sensors are mainly nodes with limited resources (exp. low power consumption). Based on the above reasons, we have designed a caching mechanism suitable for underwater environments.

In push traffic, push caching is used to determine where the content is cached and at which node the communication mode ends. In pull traffic, the decision on which nodes to cache content is called pull caching.

In the PIT and FIB tables, add a new field of cache flags. It is used to record the packet is under which communication mode. This record is benefit for distinguishing which caching mechanism to take and which records to view in subsequent packet. The caching mechanism of the above two modes is described in detail below. 


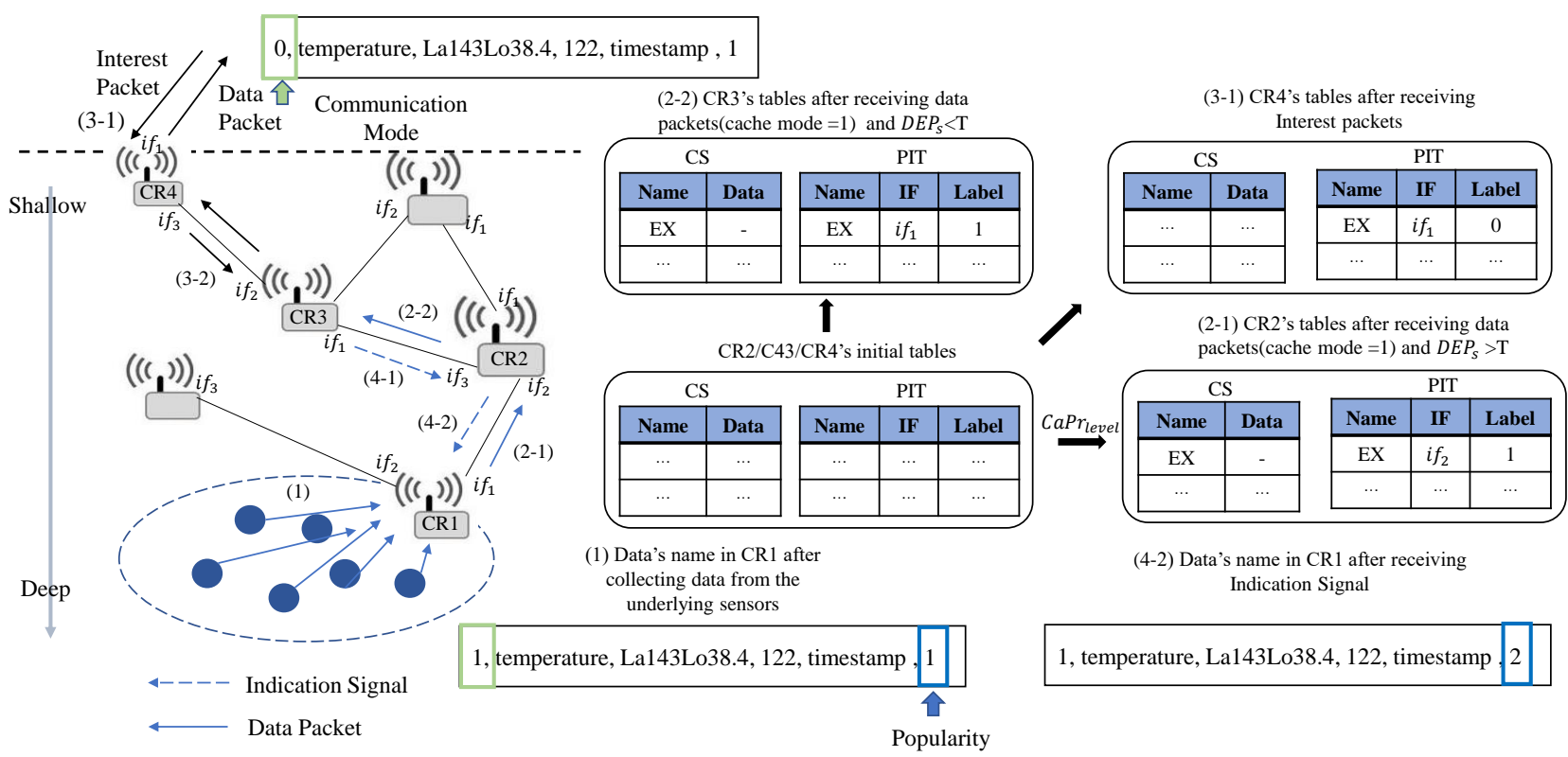

Fig. 4: The process of push traffic and push caching

A coordinated depth table (CDT) table is added for each node (except the underlying sensor node), which records the node depth reached by each interface of the current node. CDT maintains an entry for each interface. This entry records the depth of each outgoing interface and the node it reaches.

\section{A. Push Caching}

UIoT follows the 3D architecture [8], the underlying sensors form clusters, and these clusters are located at different depth levels. In each cluster, an anchor node is configured, which receives information from the underlying sensors in the cluster and communicates with other anchor sensors, surface nodes or users. The anchor node stores the data of the underlying sensor nodes in the cluster in the Content Store (CS).

The existing UIoT actively transmits all data to the users, wasting unnecessary resources and sensors' energy. On the other hand, transmitting specific data only when the user require it in ICN will increase user's waiting time. Combining the above situation, we propose a new strategy. The anchor node still needs to actively transmit data to other nodes, constantly transmitting from deep to shallow areas. Instead of transmitting data directly to the user, the designed push caching determines where to stop push traffic and caches these data at specific nodes.

After the process of push caching, users can get what they need directly from nodes that cache required content. And the memory of the bottom sensor is low, and the data caches in different upper nodes can prolong the time that the data exists. At the same time, we cannot rule out unexpected situations that occur during the transmission of data from deep to shallow, such as the content is missing or the content is not successfully transmitted. We can avoid users cannot find the required content by selectively caching content at the node during push traffic.
If the remaining battery time is low, it means that caching and subsequent transfer operations should be minimized. When a data packet reaches a certain depth during the push traffic, this mode should be stopped to avoid excessively spreading to shallow places and wasting resources. If a certain type of data in a cluster is frequently requested, the data of that type should be transmitted and cached as shallow as possible in next push traffic. Based on the above factors, depth, remaining battery life and popularity in Factor is used to measure whether the content needs to be cached at the current node and whether the push traffic to continue. Specifically, when a particular node receives a data packet from a deep place, it will calculate the $D E P_{s}$ as following:

$$
\mathrm{DEP}_{\mathrm{s}}=\omega_{1} \times \frac{\mathrm{D}_{\mathrm{n}}}{\mathrm{D}_{\mathrm{d}}}+\omega_{2} \times \mathrm{E}_{\mathrm{n}}+\omega_{3} \times \frac{\mathrm{p}}{\mathrm{T}_{\mathrm{p}}}
$$

$D_{d}$ is the depth of the content generated, and $D_{n}$ is the depth of the current node. $E_{n}$ represents the remaining battery energy, and its value is between 0 and 1. $p$ is the popularity of the content, and $T_{p}$ is threshold of popularity. $\omega_{1}, \omega_{2}, \omega_{3}$ are the weights of depth, battery remaining energy, and content popularity on influencing whether to cache.

$$
\begin{array}{ll}
\operatorname{DEP}_{\mathrm{s}}>\mathrm{T} & \text { Fowarding and Maybe Caching } \\
\operatorname{DEP}_{\mathrm{s}}<\mathrm{T} & \text { PushTraffic stops but Caching }
\end{array}
$$

$T$ is the threshold. When the cache condition is satisfied, the data packet will be transferred to other nodes, but whether the data is cached in the current node is determined by caching probability of formula (3). Otherwise, the push traffic of the data packet is terminated but cached at this node.

The main function of deep nodes is to transmit data packets to the shallow, so the cache probability of deep nodes is low. When the data packet is delivered to a shallower place, it is more possible to be cached to help users get the content faster. In this context, it is considered that the closer to the 
shallow place in push traffic, cache probability needs to be larger. Therefore, we divide nodes into different levels with different depths to calculate the cache probability of content at different nodes. The cache probability controlled by the depth level is calculated as the following formula. Where $D_{L}$ is the shallowest depth of the node's connectable interface, and M is the total number of depth level.

$$
\text { CaPr } \operatorname{Pr}_{\text {level }}= \begin{cases}\frac{1}{M} \quad & \log D_{n} \geq \log D_{d}-\frac{\log \frac{D_{d}}{D_{L}}}{M} \\ 1-\frac{\left\lceil\frac{M \times \log \frac{D_{n}}{D_{L}}}{\left.\log \frac{D_{d}}{D_{L}}-1\right\rceil}\right.}{M} & \text { else } \\ 1 & \log D_{n}<\log D_{L}+\frac{\log \frac{D_{d}}{D_{L}}}{M}\end{cases}
$$

In push caching, the cache flag is 1 , corresponding to the communication mode (push traffic) in the packet name. In this process, the PIT table hold the incoming interfaces of the data packets in the node. When a node receives an interest packet and it matches the content name of the data packet it caches, it transmits the data packet along the reverse path of the interest packet. At the same time, the node transmits the instruction signal according to the entry in the PIT table with label 1 and the same name. It reminds the underlying sensor to increase the popularity of the latter data which's content name has the same area and data type with the instruction signal. The popularity is increased by one for each received signal.

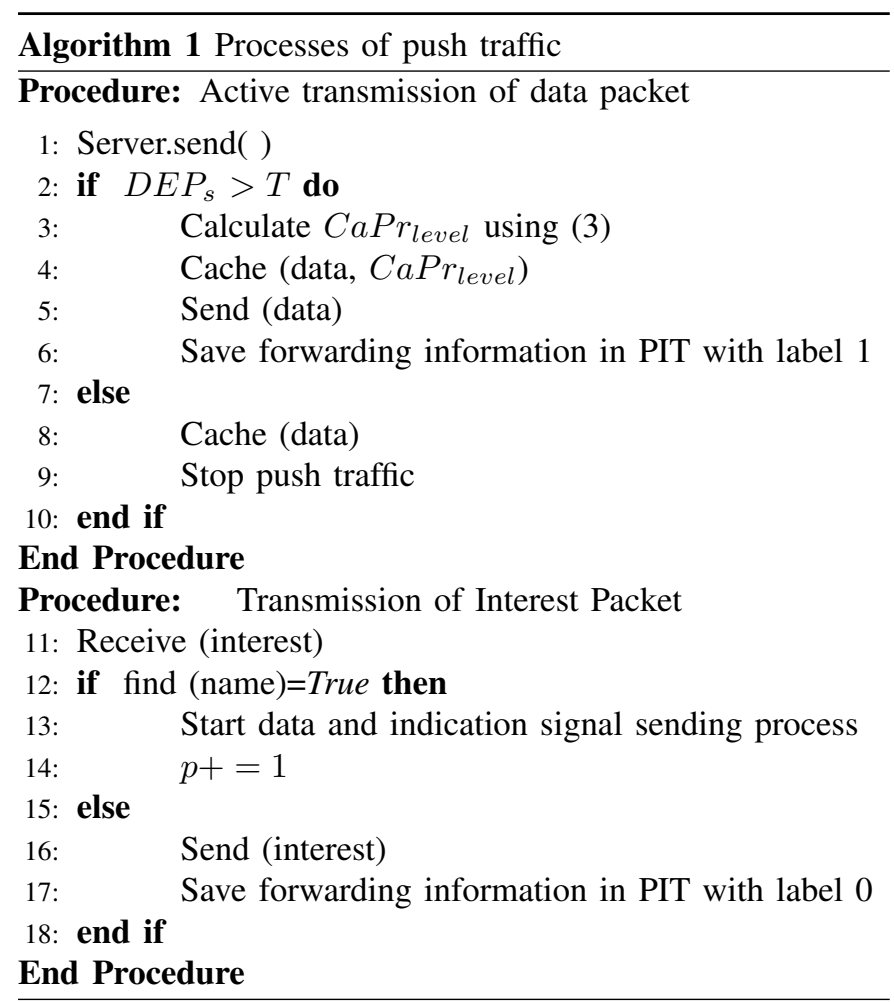

The process of push traffic in ICWSN-WDA are shown as Algorithm 1. Furthermore, we take the process of push traffic with CR1, CR2, CR3 and CR4 these four underwater sensors as an example to demonstrate how the content is cached in relatively shallow nodes and determine where the push traffic of this content stops based on Algorithm 1 and Fig. 4. It is supposed CR1 collects the content generated by the bottom sensor. After CR1 sends the content which named as EX to CR2. CR2 calculates the $D E P_{s}$ of EX at node CR2 and finds it is greater than the threshold $\mathrm{T}$, so $C a P r_{\text {level }}$ is used for caching. Then EX is transmitted to $\mathrm{CR} 3$, the $D E P_{s}$ is less than the threshold. Therefore, the push traffic ends, but CR3 caches the data. The PIT table of CR2 records the forwarding of the data packet, and label $=1$ indicates that the data packet is in the push traffic. CR4 receives the interest packet and it doesn't cache the required data, recording the entry in PIT and label $=0$. Then CR4 transmits the interest packet to CR3 in step 3-2. When CR3 receives the interest packet corresponding to EX, it transmits the data packet. The communication mode in the packet name becomes 0 . At the same time, it uses the records of the PIT tables to transmit the indication signal to the underlying sensor in step 4 , so that the popularity of the data collected in the corresponding area and data type of the EX increases by 1 to 2 .

\section{B. Pull Caching}

In the designed network, the interest packet will not be passed to the content source (the underlying sensor), but the data packet will be passed directly to the user from the node that caches the required content. In this process, it is the same as the traditional ICN process, but considering the specificity of the underwater environment, a mechanism for buffering along the path still needs to be designed.

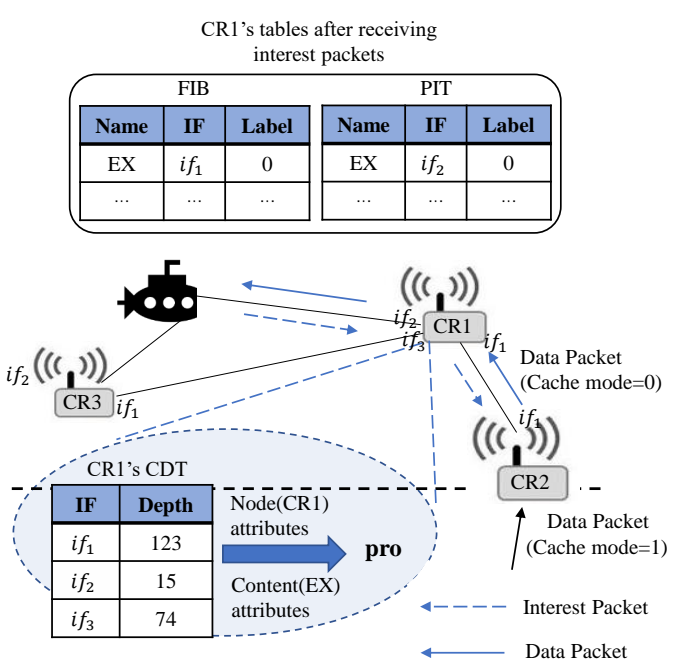

Fig. 5: The process of pull traffic and pull caching

Unlike the push cache, the process of pulling the cache is often in the area close to the water surface. Pull traffic is not necessarily transmitted from the node in the deep water to the node in the shallow water. The battery performance of the equipment in this area is significantly higher than that of equipment in the deep ocean, and the battery is easy to charge, Therefore, although the factors involved in push caching are still considered, the pull caching changes the weight of depth, battery remaining energy and popularity. 


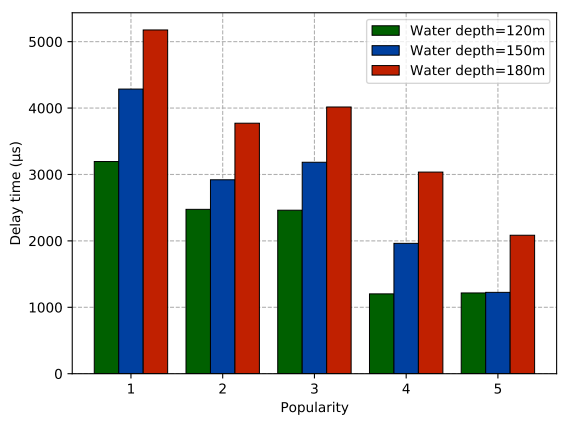

(a)

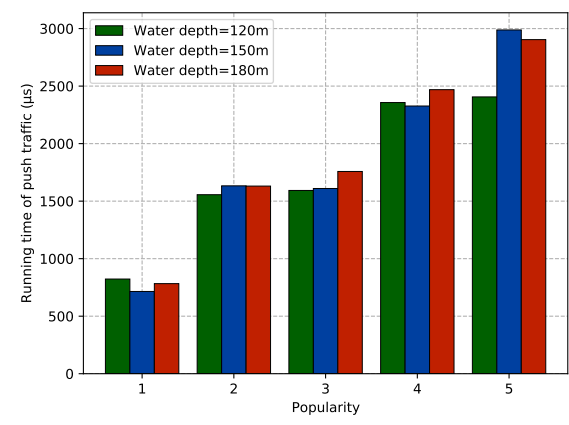

(b)

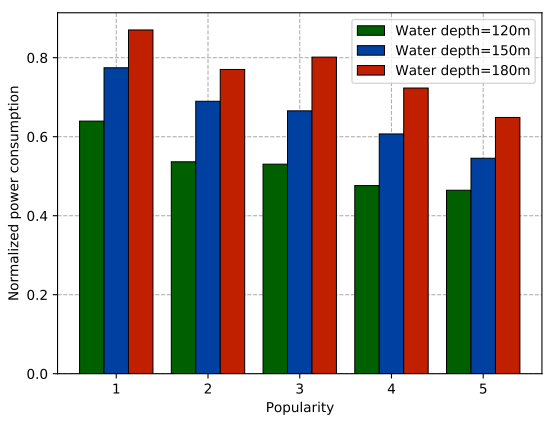

(c)

Fig. 6: (a) Delay time vs popularity; (b) Push traffic time vs popularity; (c) Power consumption vs popularity in different water depths

$$
\mathrm{DEP}_{1}=\gamma_{1} \times \frac{\mathrm{D}_{\mathrm{n}}}{\mathrm{D}_{\mathrm{d}}}+\gamma_{2} \times \mathrm{E}_{\mathrm{n}}+\gamma_{3} \times \frac{\mathrm{p}}{\mathrm{T}_{\mathrm{p}}}
$$

$\gamma_{1}, \gamma_{2}, \gamma_{3}$ are the weights of depth, battery remaining energy, and content popularity on the cache probability. The data packet in pull traffic should be forwarded to the current node's connected node no matter how deep and the cache capacity of the deep node is not as good as the shallow one. The cache probability should consider the depth of each connected node. Then use the CDT table and the current depth to calculate the cache weight of the current node.

$$
\text { CacheWeight }=\frac{D_{n} \times n}{\sum_{f_{1}}^{f_{n}} D_{i}}
$$

$D_{i}$ indicates the depth of all nodes connected to the current node and it has $n$ interfaces. Content is cached at current node with probability pro [30].

$$
\text { pro }=\text { CacheWeight } \times D E P_{l}
$$

The entire process of pull traffic and pull caching is shown in Fig. 5. The push caching actively caches the content in CR2. The user sends an interest packet to CR1, CR1 does not find the corresponding content, establishes an entry in the PIT table. The label is set to 0 . Then CR1 continues to forward interest packets to CR2. When CR2 searches for the required content, the reverse path transmits the data packet. Combining its CDT table in CR1, the node's own attributes, such as its water depth and the attributes of the content, including its generated depth and popularity, CR1 calculates pro, and use this probability to determine whether the data be cached in CR1. Then CR1 continues to forward the packet to the user.

\section{Simulation Results}

In order to evaluate the performance of the proposed ICWSN-WDA, we use the delay time and normalized power consumption. These two indicators are very important in the underwater network. It is assumed that the producer regularly pushes data, and the evaluating process follows the designed scheme behavior and matches the model assumptions. The

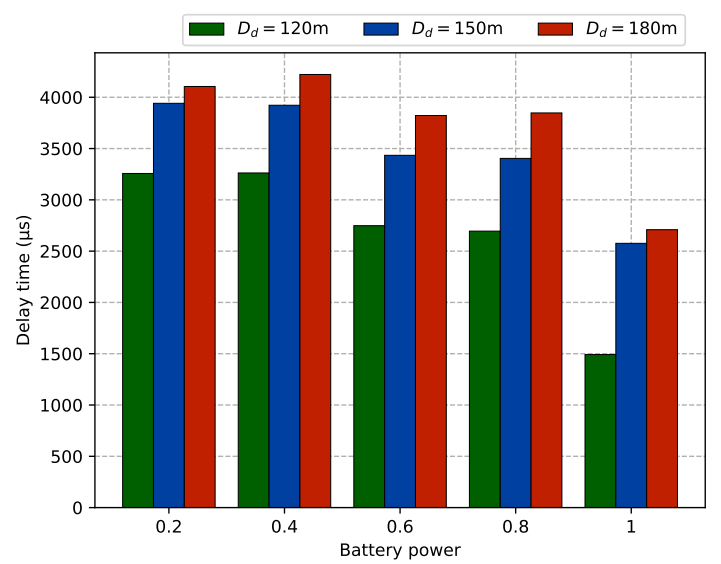

(a)

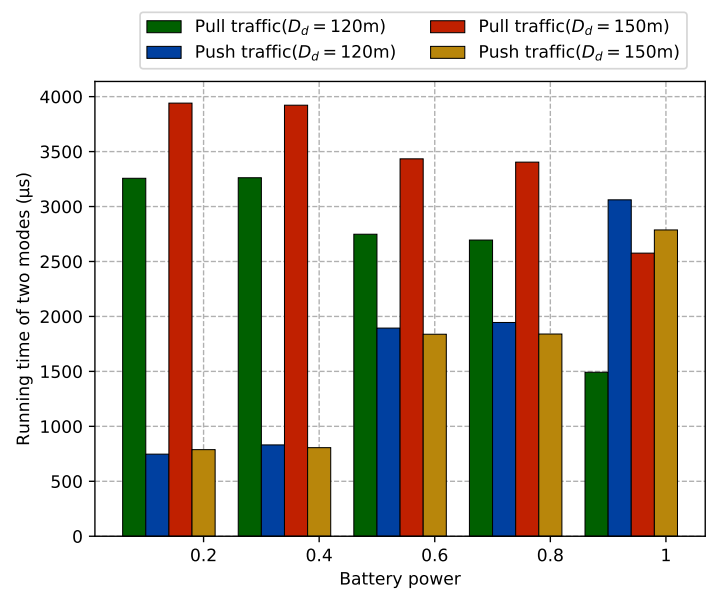

(b)

Fig. 7: (a) Delay time vs battery power in different depths; (b) Two modes' running time vs battery power

results are compared with the traditional ICN and UIoT. Figs. $6,7,8$ and 9 report the analysis and evaluation results.

Fig. 6(a) and (b) show the impact of popularity on the delay time and the running time of push traffic, respectively. The 


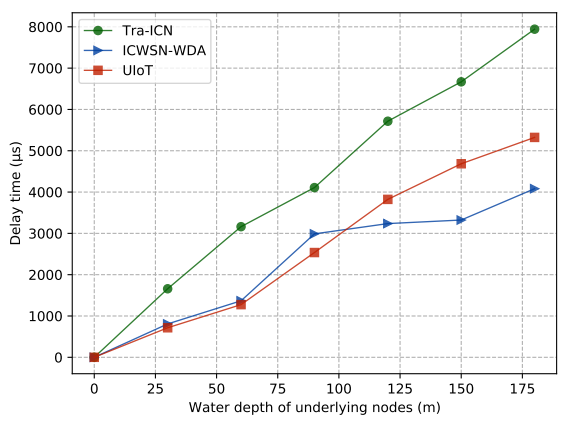

(a)

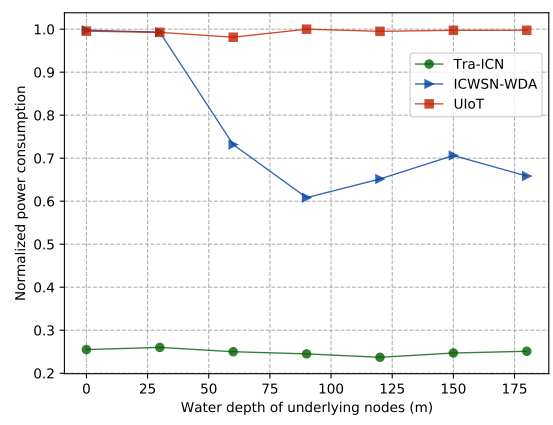

(b)

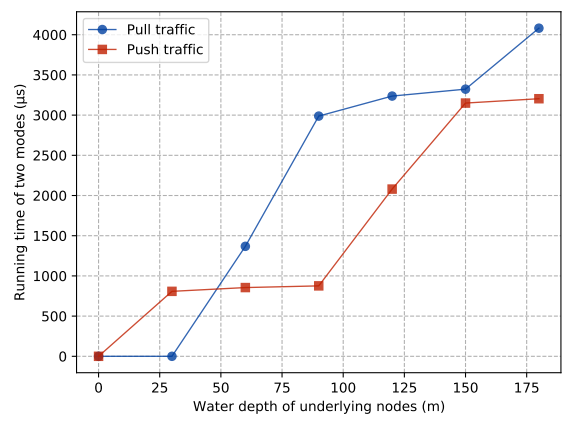

(c)

Fig. 8: (a) Delay time vs water depth in different schemes; (b) Power consumption vs water depth in different schemes; (c) Two modes' running time vs water depth

results show that as the popularity increases, the delay time decreases and the running time of push traffic increases. It means that the content is actively transmitted to shallower nodes and cached.Therefore, users can directly find the content they need at shallower nodes, reducing delay time. We investigated the impact of popularity on energy consumption, as shown in Fig. 6(c). With popularity increases, energy consumption decreases. Popular content is more likely to be cached in shallow nodes, so users request and obtain content through fewer nodes. The overall trend shows a downward trend in power consumption.

The changes of delay time caused by node battery power are shown in Fig. 7(a), considering about content generated at water depths of 120,150 , and $180 \mathrm{~m}$. It can be found that the delay time presents a phased change, and it decreases as the battery power increases. In addition, the deeper content needs longer time to be acquired by users. Compared to shallower content, nodes that cached deeper content are generally relatively deeper in push traffic. Fig. 7(b) also analyzes the impact of battery power and considers the entire communication process. Pull traffic and Push traffic show opposite trends with the increase of power. When the power is high, the content is more likely to be automatically transferred and cached to shallow nodes. Users can get the content they need directly from the shallow nodes, reducing retrieval time. Therefore, running times of two modes change at the same time.

With a focus on the ICWSN-WDA, traditional ICN and UIoT, Fig. 8(a) reports how the water depth the data collected affects the delay time, When the water depth increases, delay times of three schemes increase on the whole. And after reaching a certain water depth, the delay time of ICWSNWDA is steadily shorter than the other two schemes. Both ICWSN-WDA and UIoT directly transfer the content to the user in a shallow depth, so the delay time of them is similar in the shallow place, which demonstrated at the beginning of lines in Fig. 8(a). As the depth increases, producers need to forward through multiple nodes to communicate with users. As shown in Fig. 8(a) at $90 \mathrm{~m}$, there are a few forwarding nodes. ICWSN-WDA contains the requested-content time so that its delay time is longer than UIoT. ICWSN-WDA 's push caching allows content to be transmitted to shallower nodes and cached in shallower nodes, shortening the average latency of content requested by users. Therefore, ICWSN-WDA 's push caching makes the opposite results in $120 \mathrm{~m}$. Whether the data generated from different depths continues push traffic at current node is determined by equations in ICWSN-WDA. Therefore, data generated from different depths may stop push traffic and cache at nodes of the same depth. Namely, the data generated at the water depth of 90,120 and $150 \mathrm{~m}$ may be cached at the node of the same depth, result in the unchanged of delay times in UIoT. Traditional ICN always sends interest packets to producers, leading to the larger gap of the delay time with the other two schemes when water depth increases.

Results in Fig. 8(b) aim at comparing nodes' power consumption among three schemes. The different water depths of UIoT and traditional ICN producers have little effect on the node's power consumption, and the gap between the power consumption of them is large. Because UIoT transmits all content, all nodes are forwarding all content, while traditional ICN only forwards the required content. As the water depth increases, the power consumption shows a downward trend overall in ICWSN-WDA, while it increases between $90 \mathrm{~m}$ and $150 \mathrm{~m}$. The power consumption of the ICWSN-WDA at $30 \mathrm{~m}$ is similar to UIoT. Responding to its fluctuation in Fig. 8 (a), we can find the data generated at these water depths of 90,120 and $150 \mathrm{~m}$ may be cached at the node of the same water depth. Namely, the process of push traffic increases and unnecessary data transmits through more nodes with the water depth increases at these three depths, so power consumption increases in this interval.

The running time of two different modes in ICWSN-WDA is shown in Fig. 8(c), It can be found that both times increase with depth. However, in most cases, when one mode grows faster, the other mode changes less. Push traffic can spontaneously forward content to shallow nodes, push caching caches content and determines when the communication mode ends. It can be clearly observed that the running time of this mode increases rapidly after $60 \mathrm{~m}$. The changes of communication modes in Fig. 8(c) are similar to those in Fig. 8(a), (b). And $n$ this process, the running time changes in the unit of forwarding time between nodes. As a result, for push traffic and pull 
traffic, the running time of one communication mode increases significantly and the other one does not change significantly.

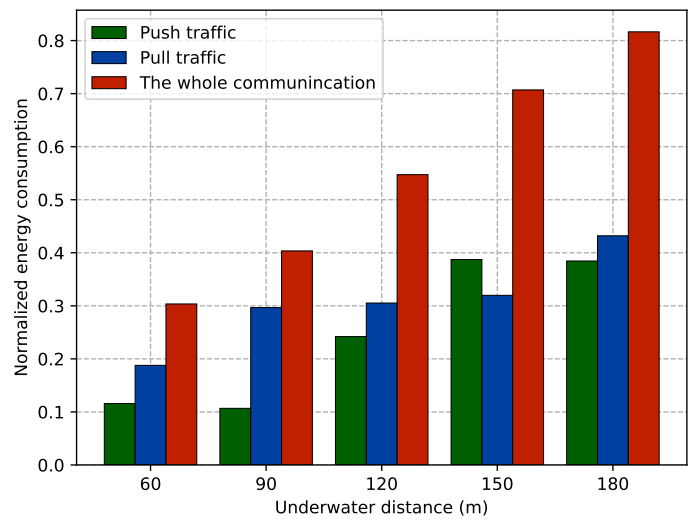

Fig. 9: Energy consumption vs underwater distance

The relationship between underwater distance and energy consumption is shown in Fig. 9. It is observed that with the increase of underwater distance, the energy consumption of push traffic and pull traffic show an overall increasing trend. For push traffic and pull traffic, the energy consumption of one communication mode increases significantly and the other one does not change obviously. As for the whole communication process, the energy consumption increases significantly with underwater distance increases.

As a conclusive remark, traditional ICN and UIoT only have good performance in one aspect, the other does not meet the requirements. And after reaching a certain water depth, the delay time of ICWSN-WDA is steadily shorter than the other two schemes. Considering comprehensively the above two factors and unique marine environment, ICWSN-WDA can better meet the needs of underwater network content transmission and other restrictions.

\section{CONCLUSION}

In this article, we designed ICWSN-WDA, an informationcentric wireless sensor networking Scheme. On the basis of improving the communication mode into a hybrid mode which includes push traffic and pull traffic, corresponding caching solutions are proposed for these two parts respectively. The caching methods take sensor power and popularity into account, and pay special attention to all aspects of water depth. The evaluation results show that ICWSN-WDA has a better performance than UIoT and the use of traditional ICN in underwater, when comprehensively consider the delay time and the energy consumption.

\section{REFERENCES}

[1] J. Catipovic, D. Brady, and S. Etchemendy, "Development of underwater acoustic modems and networks," Oceanography, vol. 6, pp. 112-119, Mar. 1993.

[2] E. M. Sozer, M. Stojanovic, J. G. Proakis, "Underwater acoustic networks," IEEE Journal of Oceanic Engineering, vol. 25, no. 1, pp. 72-83, 2000 .
[3] X. Jiang, B. Jun, G. Nan, L. Zhaogeng, "A survey on information-centric networking: Rationales designs and debates," China Commun., vol. 12 no. 7, pp. 1-12, 2015.

[4] D. D. Van and Q. Ai, "An efficient in-network caching decision algorithm for Internet of Things," Int. J. Commun. Syst., vol. 31, no. 8, 2018 , Art. no. e3521.

[5] J. Wu, M. Dong, K. Ota, J. Li, W. Yang, "Application-Aware Consensus Management for Software-defined Intelligent Blockchain in IoT," IEEE Network, vol. 34, no. 1, pp. 69-75, 2020.

[6] H. Liang, J. Wu, S. Mumtaz, J. Li, X. Lin and M. Wen, "MBID: MicroBlockchain based Geographical Dynamic Intrusion Detection for V2X," IEEE Communications Magazine, vol. 57, no. 10, pp. 77-83, 2019.

[7] X. Lin, J. Wu, S. Mumtaz, S. Garg, J. Li, and M. Guizani, "Blockchainbased On-demand Computing Resource Trading in IoV-Assisted Smart City," IEEE Transactions on Emerging Topics in Computing,, DOI: 10.1109/TETC.2020.2971831, 2020

[8] K. Wang, J. Wu, X. Zheng, A. Jolfaei, J. Li, D. Yu, "Leveraging Energy Function Virtualization with Game Theory for FaultTolerant Smart Grid," IEEE Transactions on Industrial Informatics, DoI: 10.1109/TII.2020.2971584, 2020.

[9] X. Lin, J. Wu, A. K. Bashir, J. Li, W. Yang, and J. Piran, "Blockchain-Based Incentive Energy-Knowledge Trading in IoT: Joint Power Transfer and AI Design," IEEE Internet of Things Journal, DOI: 10.1109/JIOT.2020.3024246, 2020.

[10] T. Qiu, Z. Zhao, T. Zhang, C. Chen and C. L. P. Chen, "Underwater Internet of Things in smart ocean: System architecture and open issues", IEEE Trans. Ind. Informat., vol. 16, no. 7, pp. 4297-4307, Jul. 2020

[11] H. Luo, K. Wu, R. Ruby, Y. Liang, Z. Guo and L. M. Ni, "Softwaredefined architectures and technologies for underwater wireless sensor networks: A survey", IEEE Commun. Surveys Tuts., vol. 20, no. 4, pp. 2855-2888, 4th Quart. 2018.

[12] Yishan Su, Lin Zhang, Yun Li, Xing Yao, "A Glider-Assist Routing Protocol for Underwater Acoustic Networks With Trajectory Prediction Methods", Access IEEE, vol. 8, pp. 154560-154572, 2020.

[13] Vinayak Khajuria, Manjot Kaur, "Underwater Wireless Sensor Network: Architecture, Applications and Challenges," inProc. 2nd International Conference on Trends in Electronics and Informatics, pp:939-944, 2018.

[14] Named Data Networking (NDN) Project. Accessed: Jun. 10, 2015. [Online]. Available: http://named-data.net/.

[15] Pursuing a Pub/Sub Internet-FP7 Project Pursuit. Accessed: Jun. 12, 2015. [Online]. Available: http://www.fp7-pursuit.eu/ PursuitWeb/.

[16] Mobilityfirst Future Internet Architecture Project. Accessed: Apr. 24 2015. [Online]. Available: http://mobilityfirst.winlab.rutgers.edu/.

[17] Green icn Architecture and Applications of Green Information Centric Networking. Accessed: Jul. 30, 2016. [Online]. Available: http://www.greenicn.org/.

[18] X. Lin, J. Li, J. Wu, H. Liang, W. Yang, "Making Knowledge Tradable in Edge-AI Enabled IoT: A Consortium Blockchain-based Efficient and Incentive Approach," IEEE Transactions on Industrial Informatics, vol. 15, no. 12, pp. 6367-6378, 2019.

[19] M. Amadeo, C. Campolo, A. Iera, and A. Molinaro, "Named data networking for IoT: An architectural perspective," in Proc. Eur. Conf. Netw. Commun. (EuCNC) 2014, pp. 1-5.

[20] R. Tourani, S. Misra, T. Mick, S. Brahma, M. Biswal, D. Ameme, "icens: An information-centric smart grid network architecture," 2016 IEEE International Conference on Smart Grid Communications (SmartGridComm), pp. 417-422, Nov 2016.

[21] J. Wu, M. Dong, K. Ota, J. Li, W. Yang and M. Wang, "Fog Computing enabled Cognitive Network Function Virtualization for InformationCentric Future Internet," IEEE Communications Magazine, vol. 57, no. 7, pp. 48-54, 2019.

[22] Z. Yan, S. Zeadally, Y.-J. Park, "A novel vehicular information network architecture based on named data networking (NDN)," IEEE Internet Things J., vol. 1, no. 6, pp. 525-532, Dec. 2014.

[23] J. Wu, M. Dong, K. Ota, J. Li, Z. Guan, "FCSS: Fog-Computing-based Content-Aware Filtering for Security Services in Information-Centric Social Networks," IEEE Transactions on Emerging Topics in Computing, vol. 7, no. 4, pp. 553-564, 2019

[24] Q. Guan, F. Ji, Y. Liu, H. Yu and W. Chen, "Distance-Vector-Based opportunistic routing for underwater acoustic sensor networks," IEEE Internet Things J., vol. 6, no. 2, pp. 3831-3839, Apr. 2019.

[25] I. Akyildiz, D. Pompili, and T. Melodia, "Underwater acoustic sensor networks: Research challenges," Elsevier Ad Hoc Networks, vol. 3, no. 3, pp. 257-279, 2005.

[26] J. Heidemann, W. Ye, J. Willis, A. Syed, and Y. Li, "Research challenges and applications for underwater sensor networking," in Proc. IEEE 
Wireless Communications of Networking Conference (WCNC), Las Vegas, USA, Apr 2006.

[27] E. M. Sozer, M. Stojanovic, and J. G. Proakis, "Underwater acoustic networks," IEEE J. Oceanic Eng., vol. 25, no. 1, pp. 72-83, Jan. 2000.

[28] Youngjun Jo, Jungyoung Bae, Hyunjae Shin, Heungwoo Nam, Saeyoung Ahn,. and Sunshin An, "The architecture of surface gateway for underwayer acoustic networks", 4th IEEE/IFIP International workshop on ubiquitous Underwater Sensor Network, Hong Kong, China, 2010.

[29] H. Ghafoor and Y. Noh, "An overview of next-generation underwater target detection and tracking: An integrated underwater architecture," IEEE Access, vol. 7, pp. 98841-98853, 2019.

[30] I. Psaras, W. K. Chai, and G. Pavlou, "In-network cache management and resource allocation for information-centric networks," IEEE Trans. Parallel Distrib. Syst., vol. 25, no. 11, pp. 2920-2931, Dec. 2013.

[31] Jing, Shusen, Joseph Hall, Yahong Rosa Zheng, and Chengshan Xiao. "Signal Detection for Underwater IoT Devices with Long and Sparse Channels." IEEE Internet of Things Journal, vol. 7, no. 8, pp: $6664-$ 6675,2020

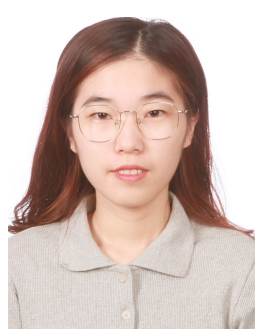

Jiana Li received the B.S. degree from School of Information Security in Hunan University, Hunan, China, in 2019. Now, she is a candidate M.S. at School of Electronic Information and Electrical Engineering, Shanghai Jiao Tong University, Shanghai, China. Her research interests are focusing on Information-Centric Network (ICN), Underwater Wireless Sensor Networking (UWSN), etc.

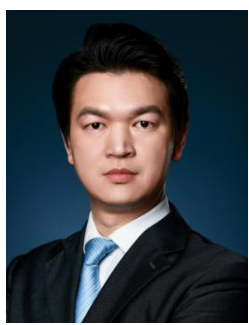

Jun Wu received the Ph.D. degree in information and telecommunication studies from Waseda University, Japan, in 2011. He was a Post-Doctoral Researcher with the Research Institute for Secure Systems, National Institute of Advanced Industrial Science and Technology (AIST), Japan, from 2011 to 2012. He was a Researcher with the Global Information and Telecommunication Institute, Waseda University, Japan, from 2011 to 2013. He is currently a professor of School of Electronic Information and Electrical Engineering, Shanghai Jiao Tong University, China. He is also the vice dean of Institute of Cyber Science and Technology and vice director of National Engineering Laboratory for Information Content Analysis Technology, Shanghai Jiao Tong University, China. He is the chair of IEEE P21451-1-5 Standard Working Group. He has hosted and participated in a lot of research projects including National Natural Science Foundation of China (NFSC), National 863 Plan and 973 Plan of China, Japan Society of the Promotion of Science Projects (JSPS), etc. His research interests include the advanced computing, communications and security techniques of software-defined networks (SDN), information-centric networks (ICN), Internet of Things (IoT), 5G/6G, molecular communication, etc., where he has published more than 150 refereed papers. He has been the Track Chair of VTC 2019, VTC 2020 and the TPC Member of more than ten international conferences including ICC, GLOBECOM, etc. He has been a Guest Editor of IEEE Transactions on Industrial Informatics, IEEE Sensors Journal, Sensors. He is an Associate Editor of the IEEE Access, IEEE Networking Letters.

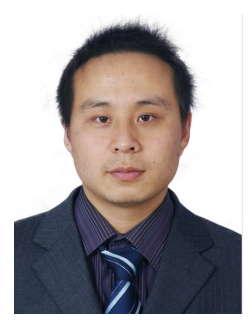

Changlian $\mathbf{L i}$ is deputy chief engineer of Intelligent Network Design Institute, China Information Consulting \& Designing Institute Co., Ltd.. He got his MS. degree in computer software and theory from Northwestern Polytechnical University in 2007. Currently, he engages in top network security layer program of telecom operators, the preparation of security standards, the independent research and development of security products and operation support. He developed China Unicom Cloud Shield DDoS protection, DNS protection and other domestic heavyweights of Network security products. He participated in the research work of the major topic of network security, the Ministry of Science and Technology.

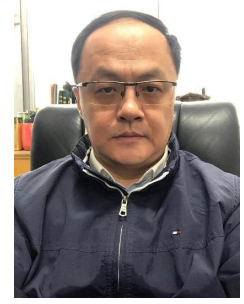

Wu Yang received the Ph.D. degree in computer system architecture specialty from the Computer Science and Technology School, Harbin Institute of Technology. He is currently a Professor and a Doctoral Supervisor with Harbin Engineering University. His main research interests include wireless sensor network, peer-to-peer network, and information security. He is a member of ACM and a Senior Member of CCF.

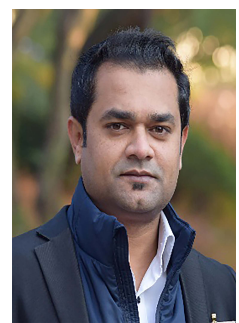

Ali Kashif Bashir is a Senior Lecturer/Associate Professor and Course Leader of BSc (H) Computer Forensics and Security at the Department of Computing and Mathematics, Manchester Metropolitan University, United Kingdom. He is also with School of Electrical Engineering and Computer Science, National University of Science and Technology, Islamabad (NUST) as an Adjunct Professor and School of Information and Communication Engineering, University of Electronics Science and Technology of China (UESTC) as an Affiliated Professor and Chief Advisor of Visual Intelligence Research Center, UESTC. He is a senior member of IEEE, member of IEEE Industrial Electronic Society, member of ACM, and Distinguished Speaker of ACM. His past assignments include Associate Professor of ICT, University of the Faroe Islands, Denmark; Osaka University, Japan; Nara National College of Technology, Japan; the National Fusion Research Institute, South Korea; Southern Power Company Ltd., South Korea, and the Seoul Metropolitan Government, South Korea. $\mathrm{He}$ has worked on several research and industrial projects of South Korean, Japanese and European agencies and Government Ministries. He received his $\mathrm{Ph} . \mathrm{D}$. in computer science and engineering from Korea University South Korea. He has authored over 180 research articles; received funding as PI and Co-PI from research bodies of South Korea, Japan, EU, UK and Middle East; supervising/co-supervising several graduate (MS and PhD) students. His research interests include internet of things, wireless networks, distributed systems, network/cyber security, network function virtualization, machine learning, etc. He is serving as the Editor-in-chief of the IEEE FUTURE DIRECTIONS NEWSLETTER. He is also serving as area editor of KSII Transactions on Internet and Information Systems; associate editor of IEEE Internet of Things Magazine, IEEE Access, Peer J Computer Science, IET Quantum Computing, Journal of Plant Disease and Protection. He is leading many conferences as a chair (program, publicity, and track) and had organized workshops in flagship conferences like IEEE Infocom, IEEE Globecom, IEEE Mobicom, etc.

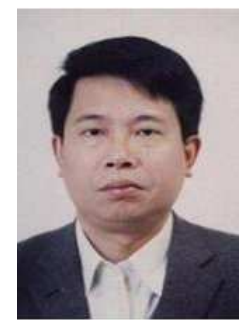

Jianhua $\mathbf{L i}$ is a professor/Ph.D. supervisor and the dean of School of Information Security Engineering, Shanghai Jiao Tong University, Shanghai, China. He got his BS, MS and Ph.D. degrees from Shanghai Jiao Tong University, in 1986, 1991 and 1998, respectively. He was the chief expert in the information security committee experts of National High Technology Research and Development Program of China (863 Program) of China. He is the member of the committee of information security area of the state 10th five-year plan of China. Also, he is a committee expert of China State Secrecy Bureau and Shanghai Secrecy Bureau. He was the leader of more than 30 state/province projects of China, and published more than 200 papers. He published 6 books and has about 20 patents. He made 3 standards and has 5 software copyrights. He got the Second Prize of National Technology Progress Award of China in 2005. He got the First Prize of National Technology Progress Award of Shanghai in 2003 and 2004, and he got two First Prize of National Technology Progress Awards of Shanghai in 2004. His research interests include cyberspace security, Next Generation Networks, etc.

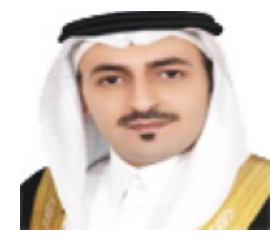

Yasser D. Al-Otaibi received the Ph.D. degree in information systems from Griffith University, Brisbane, QLD, Australia, in 2018. He is currently an Assistant Professor with the Department of Information Systems, College of Computing and Information Technology in Rabigh, King Abdulaziz University, Rabigh, Saudi Arabia. His current research interests include the areas of adoption and acceptance of ITbased innovations by individuals and issues related to information systems continuance. 